\title{
The cleavage of HuR interferes with its transportin-2- mediated nuclear import and promotes muscle fiber formation
}

\author{
P Beauchamp ${ }^{1,3}$, C Nassif ${ }^{1,3}$, S Hillock ${ }^{2}, \mathrm{~K}$ van der Giessen ${ }^{1}$, C von Roretz ${ }^{1}$, BJ Jasmin ${ }^{2}$ and I-E Gallouzi ${ }^{\star, 1}$
}

\begin{abstract}
Although the function of posttranscriptional processes in regulating the expression of genes involved in muscle fiber formation (myogenesis) is well accepted, the mechanisms by which these effects are mediated remain elusive. Here, we uncover such a mechanism and show that during myogenesis, a fraction of the posttranscriptional regulator human antigen $R(H u R)$ is cleaved in a caspase-dependent manner in both cell culture and animal models. Disruption of caspase activity in cultured myoblasts or knocking out the caspase-3 gene in mice significantly reduced HuR cleavage and the cytoplasmic accumulation of HuR in muscle fibers. The non-cleavable isoform of HuR, HuRD226A, failed to reestablish the myogenic potential of HuR-depleted myoblasts. HuR cleavage generates two fragments: HuR-cleavage product 1 (HuR-CP1) (24 kDa) and HuR-CP2 (8 kDa). Here, we show that one of these fragments (HuR-CP1) binds to the HuR import factor transportin-2 (TRN2) allowing HuR to accumulate in the cytoplasm. As this cytoplasmic accumulation is required for the promyogenic function of HuR, our data support a model, whereby during the transition phase from myoblasts to myotubes, a proportion of HuR is cleaved to generate HuR-CP1. By interfering with the TRN2-mediated import of HuR, this CP helps non-cleaved HuR accumulate in the cytoplasm thus promoting myogenesis.
\end{abstract}

Cell Death and Differentiation (2010) 17, 1588-1599; doi:10.1038/cdd.2010.34; published online 9 April 2010

One of the main features of muscle tissue is its ability to repair itself upon injury by initiating a process known as myogenesis, which replaces damaged muscle fibers. ${ }^{1}$ Myogenesis involves the sequential activation of myogenic regulatory factor (MRF) genes, ${ }^{2}$ which trigger the fusion of embryonic muscle cells (myoblasts) to form muscle fibers (myotubes). ${ }^{1}$ MRFs such as MyoD, myogenin, myogenic factor 5 , and MRF4 belong to a family of transcription factors that are responsible for stimulating the expression of promyogenic genes. ${ }^{2}$ Upon activation of myogenesis, MyoD is the first MRF to be upregulated and continues to be expressed in myotubes. MyoD then collaborates with myogenin to promote the fusion of myobasts, leading to muscle fiber formation. ${ }^{1,3}$

Although the signal transduction pathways that activate the expression of MRFs at the transcriptional level are well described, transcription alone is not sufficient to maintain the high levels of their respective proteins required during the myogenic process. ${ }^{1,3,4}$ Others and we have suggested that posttranscriptional events are important for muscle development and maintenance. ${ }^{5,6}$ Messages encoding MyoD and myogenin proteins contain cis-elements, known as AU-rich elements (AREs), in their $3^{\prime}$ untranslated regions ( $3^{\prime}$ UTRs). AREs modulate the turnover of several mRNAs in muscle and other cell types by mediating their association with
RNA-binding proteins such as the human antigen $\mathrm{R}(\mathrm{HuR})$ protein. $^{7,8}$ In fact, upon the induction of myogenesis, HuR associates with the $3^{\prime}$ UTR of acetylcholinesterase, MyoD, and myogenin mRNAs, leading to their stabilization. ${ }^{5,6,9}$ Therefore, posttranscriptional regulatory events involving interactions between AREs in myogenic mRNAs and HuR protein have crucial functions during myogenesis.

Recent observations have indicated that the promyogenic function of HuR involves its import receptor transportin-2 (TRN2). At the initial steps of myogenesis, HuR is localized to the nucleus, whereas during cell fusion and muscle fiber formation, HuR accumulates in the cytoplasm, ${ }^{5}$ coinciding with the interruption of the association of HuR with TRN2. ${ }^{10}$ Both the dissociation of the HuR/TRN2 complex and the depletion of TRN2 expression stabilize $M y o D$ and myogenin mRNAs and promote myoblast differentiation. ${ }^{10}$

Recently, we observed that the rapid cytoplasmic accumulation of HuR also occurs in cells undergoing apoptosis in response to several stresses. ${ }^{11}$ Indeed, under lethal conditions, cells initiate the caspase-3/7-mediated cleavage of HuR at the Asp (D) 226 residue, generating two cleavage products (CPs): HuR-CP1 (24 kDa) and HuR-CP2 (8 kDa). In HeLa cells, HuR-CP2, but not HuR-CP1, associates with the apoptosome activator PHAPI to promote apoptosis, whereas

\footnotetext{
${ }^{1}$ Biochemistry Department, McGill University, Rosalind and Moris Goodman Cancer Center, Montreal, Quebec, Canada and ${ }^{2}$ Department of Cellular and Molecular Medicine and Centre for Neuromuscular Disease, Faculty of Medicine, University of Ottawa, Ottawa, Ontario, Canada

${ }^{*}$ Corresponding author: I-E Gallouzi, Department of Biochemistry, McGill University, 3655 Promenade Sire William Osler, McIntyre Building room 906A, Montreal, Quebec, Canada H3G 1Y6. Tel: + 1514398 4537; Fax: + 1514398 7384; E-mail: imed.gallouzi@ mcgill.ca

${ }^{3}$ These authors contributed equally to this work.

Keywords: HuR; muscle cell differentiation; caspases; transportin-2; protein import; mRNA stability

Abbreviations: HuR, human antigen R; TRN2, transportin-2; MRFs, myogenic regulatory factors; AREs, AU-rich elements; HuR-CP, HuR cleavage product; caspase-CP, caspase cleavage product; AP, antennapedia; GFP, green fluorescence protein; Rec, recombinant; GST, gluthation S-transferase; RRM, RNA recognition motif; ActD, actinomycin D

Received 21.10.09; revised 19.2.10; accepted 22.2.10; Edited by R De Maria; published online 09.4.10
} 
overexpression of the non-cleavable isoform of HuR, HuR$\mathrm{D} 226 \mathrm{~A}$, delayed this fatal outcome. Interestingly, several studies have shown the implication of important apoptotic players such as caspase- 3 and p53 in regulating myogenesis. ${ }^{12-14}$ It has been shown that caspase-3 enhances the rate of myofiber formation by specifically cleaving the promyogenic mammalian steril 20 -like kinase, ${ }^{3}$ leading to its activation. ${ }^{12}$ The fact that caspase- 3 promotes myogenesis 12,15 raises the possibility that caspase-mediated cleavage could be part of the regulatory mechanisms modulating the promyogenic function of HuR.

In this study, we tested this possibility and present data showing that the caspase-mediated cleavage of HuR is crucial for myogenesis. We showed that this caspase activity generates HuR-CP1, which interferes with the TRN2mediated import of full-length HuR into the nucleus, thereby promoting muscle fiber formation.

\section{Results}

HuR is cleaved in a caspase-dependent manner, both in cultured $\mathrm{C2C12}$ cells and in mice, during myogenesis. To assess whether HuR is cleaved by caspases during myogenesis, we followed HuR expression throughout the differentiation of a well-established murine myogenic cell line, C2C12. ${ }^{16,17}$ To ensure that any cleavage of HuR was not the result of contamination with apoptotic muscle cells, ${ }^{18}$ the $\mathrm{C} 2 \mathrm{C} 12$ cells, differentiated or not, were washed several times to remove the small number of nonadherent dead cells that are normally seen during the myogenic process. ${ }^{15}$ The $4^{\prime}, 6$-diamidino-2-phenylindole (DAPI) staining of the remaining adherent cells showed that $>98 \%$ of them had a normal nuclear morphology (Supplementary Figure S1). Western blot analysis of the differentiating $\mathrm{C} 2 \mathrm{C} 12$ cells with the anti-HuR monoclonal antibody ${ }^{19}$ detected full-length HuR and a second band at $24 \mathrm{kDa}$, which began appearing at day 1 of myogenesis with a maximum intensity at day 4 (Figure $1 a$ and $b$ ). The size of this band corresponds to the size of the HuR cleavage product 1 (HuR-CP1) that was earlier seen in HeLa cells during caspase-induced apoptosis. ${ }^{11}$ We also observed that this additional band reached its maximum levels at day 4 of the differentiation process, a time during which muscle cells generated, as expected, ${ }^{15}$ the active isoform of caspase-3, caspase-3-CP (Figure 1a and b).

Next, we assessed whether the HuR-CP1 band would also be detected during the regeneration of skeletal muscle in mice. The tibialis anterior (TA) muscle of 4-week-old caspase$3+/+$ or $-/-$ mice was treated with $10 \mu \mathrm{M}$ cardiotoxin, which induces muscle degeneration followed by regeneration ${ }^{20,21}$ (Supplementary Figure S2). Following the expression of HuR during muscle regeneration, we detected the same $24 \mathrm{kDa}$ band seen in differentiating $\mathrm{C} 2 \mathrm{C} 12$ cells (Figure 1c, lanes 1-4 and d). This $24 \mathrm{kDa}$ band was absent in differentiating $\mathrm{C} 2 \mathrm{C} 12$ cells that were exposed to the caspase inhibitor $z V A D^{22}$ (Figure 1e) and was significantly reduced in the TA muscle of caspase-3 - /- mice (Figure 1c, lanes 5-8 and d). The same reduction in the cleavage of HuR was also observed in the extensor digitorum longus (EDL) muscle of caspase-3 -/- mice (data not shown). Although as expected, ${ }^{5} \mathrm{HuR}$ level was very low in untreated TA muscles (Figure 1c, lanes 1 and 5), the decreased HuR-CP1 levels observed in cardiotoxintreated TA muscles of caspase-3 -/- mice or in zVADtreated myotubes (Figure 1c and e) correlated with a drastic reduction in myogenin protein levels. We then determined how much of total HuR is cleaved during myogenesis in our cell culture and animal models. Using the ImageQuant software and by calculating the ratio of band intensities corresponding to HuR-CP1 over total HuR levels, we determined that in differentiated muscle fibers, HuR-CP1 represents $\sim 11 \%$ of total HuR (Figure $1 \mathrm{~b}$ ) and that this number is slightly higher $(\sim 15 \%)$ in mice (Figure 1d). Therefore, these observations indicate that during muscle fiber formation, in both cultured cells and muscle tissue from mice, one or more members of the caspase family are responsible for the cleavage of $10-15 \%$ of total HuR.

Our earlier study indicated that although the C-terminal region of the human HuR harbors three potential cleavage sites (D226, D254, and D256), only the D226 residue is targeted by caspases- 3 and -7 in HeLa cells undergoing apoptosis. ${ }^{11}$ As these three $D$ residues are also conserved in murine HuR (Supplementary Figure S3), we investigated whether any of these aspartates are the sites of cleavage by caspases in muscle cells. Each $D$ residue was replaced by alanine $(A)$ and their expression in differentiating $\mathrm{C} 2 \mathrm{C} 12$ cells was determined (Figure $2 a$ and b). Western blot using the anti-green fluorescence protein (anti-GFP) antibody showed that cleavage occurs at the same level as GFP-tagged wild-type HuR for the GFP-HuRD254A and GFP-HuRD256A mutants, but is very week for the mutant GFP-HuRD226A (Figure 2b, compare lane 4 with lanes 1, 2, and 3 and see Figure $1 a$ and $b$ ). Using the same calculation method described above (Figure 1), we observed that the cleavage efficiency of GFP-HuRD226A is 10-fold less then the cleavage efficiency of the other HuR isoforms. These observations indicate that in muscle cells, the $\mathrm{D} 226$ residue represents the main, but not the unique, cleavage site for HuR that is targeted by caspases during the differentiation process.

Caspase-mediated cleavage of HuR is needed for its promoyogenic function. We next investigated the impact of the D226A mutation on the promyogenic function of HuR. To achieve this, we assessed whether HuR or HuRD226A would reestablish myogenesis in HuR-depleted $\mathrm{C} 2 \mathrm{C} 12$ cells. ${ }^{6}$ To ensure high uptake efficiency of wtHuR and HuRD226A in muscle cells, these isoforms were conjugated to the antennapedia (AP) cell-permeable peptide ${ }^{6}$ (Supplementary Figure S4) and the chimeras were then introduced into HuR-depleted C2C12 myoblasts. Our data show that all the AP-conjugated HuR isoforms were taken up by the cell with the same efficiency and remained stable inside the cell for $>8 \mathrm{~h}$ (Supplementary Figure S4). The effect of AP-HuR and AP-HuD226A on myogenesis was assessed by phase contrast (Figure $3 \mathrm{~b}$ ) and by immunofluorescence experiments in which we followed the expression of the myoglobin protein, a known marker of muscle differentiation (Figure 3c). ${ }^{6}$ Myoglobin expression was also used to determine the fusion index (Figure 3d), 
a

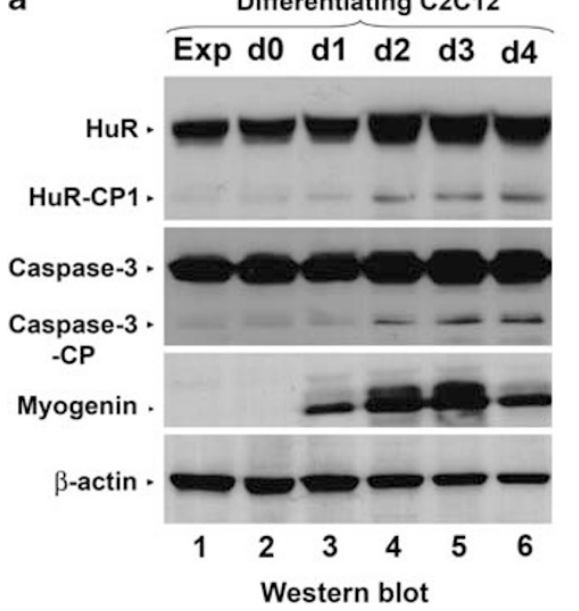

b

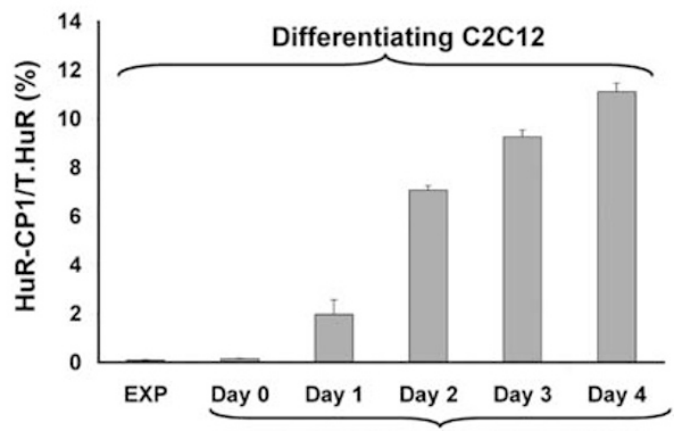

Days post differentiation initiation

C

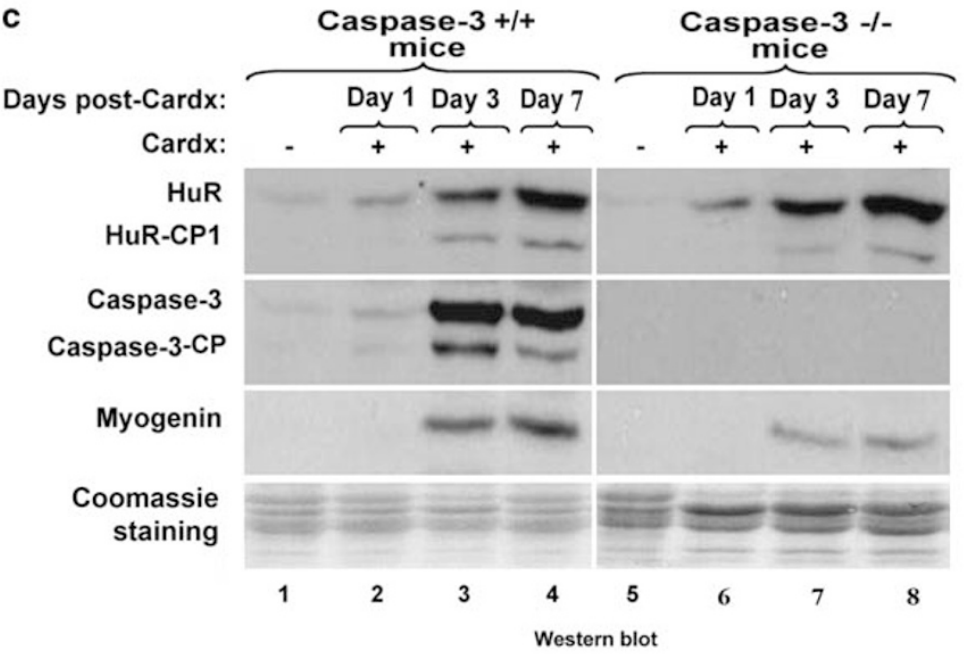

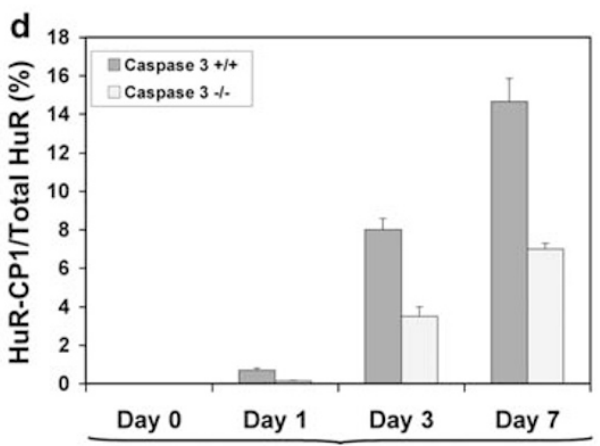

Days post Cardiotoxin treatments of TA muscle in mice e

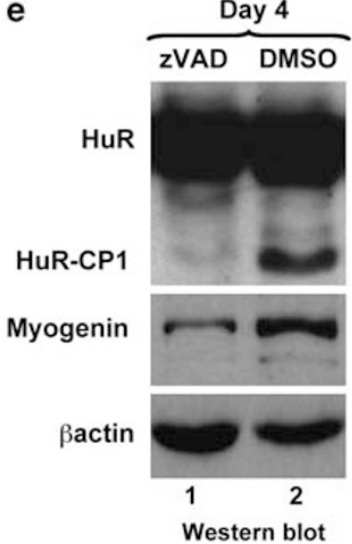

Figure 1 HuR-CP1 is detected at late stages of myogenesis both in differentiating $\mathrm{C} 2 \mathrm{C} 12$ cells and in regenerating muscle fibers in mice. (a) Western blot analyses were performed using $100 \mu \mathrm{g}$ of total protein extracts from exponentially growing (Exp) and differentiating C2C12 cells days (d) $0-4$. The blot was probed with the monoclonal anti$\mathrm{HuR}(3 \mathrm{~A} 2)$, caspase-3, myogenin, and $\beta$-actin antibodies. (b) The levels of HuR-CP1 and total HuR were quantified using the ImageQuant software. Levels were then standardized against $\beta$-actin for each time point and plotted as the percentage $+I$ - the standard error of the mean (S.E.M.) of three independent experiments. (c) Western blot analysis was performed using $80 \mu \mathrm{g}$ of total protein extracts from regenerating TA muscles of two wild-type (WT) and caspase-3 - / - mice (Casp3tm1 Flv/J mice) for each time point. Regeneration was induced by injecting $10 \mu \mathrm{M}$ cardiotoxin directly into TA muscles. Blots were probed with antibodies against HuR, caspase-3, and Myogenin. TA muscles were isolated from untreated $(-)$ and at days 1,3 , and 7 postinjection. (d) The levels of HuR-CP1 in (c) were calculated as described in (b) except that they were standardized against the band intensities of the coomassie blue staining for each sample. Data are represented as $+/-$ S.E.M. of three independent experiments. (e) C2C12 myotubes on day 3 of differentiation were treated with the pan-caspase inhibitor ZVAD for $16 \mathrm{~h}$, and total cell extracts were prepared on day 4 of differentiation for western blotting to probe for HuR, myogenin, and $\beta$-actin 


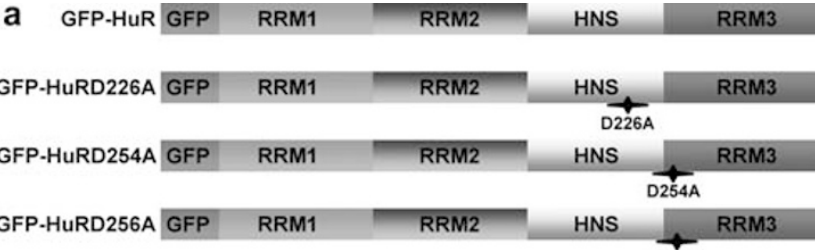

b

\begin{tabular}{|c|c|c|}
\hline \multicolumn{3}{|c|}{ Vestern blot/aHuR } \\
\hline \multicolumn{3}{|c|}{ Day 3 of differentiation } \\
\hline $\begin{array}{c}\text { HuR } \\
\text { D226A }\end{array}$ & $\begin{array}{c}\text { HuR } \\
\text { D254A }\end{array}$ & $\begin{array}{c}\text { HuR } \\
\text { D256A }\end{array}$ \\
\hline
\end{tabular}

$\alpha$ GFP $\left\{\begin{array}{l}\text { HuR/muts } \\ \text { HuR-CP1 }\end{array}\right.$

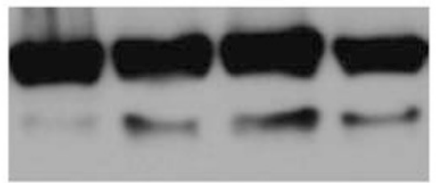

End-HuR

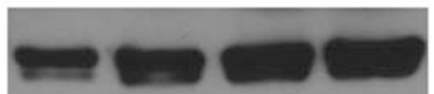

$\beta$-actin

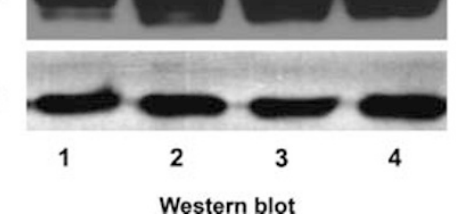

Figure 2 The D22A residue represents the major site that is targeted by caspases to cleave HuR during the differentiation of $\mathrm{C} 2 \mathrm{C} 12$ muscle cells. (a) Schematic representation of GFP-tagged wild-type HuR (GFP-HuR) and GFPtagged HuR mutants (GFP-HuRD226A, GFP-HuRD254A, GFP-HuRD256A). (b) $\mathrm{C} 2 \mathrm{C} 12$ cells were transfected with GFP-HuR, -HuRD254A, -HuRD256A, and -HuRD226A mutants as indicated. The cells were induced for differentiation for 3 days, then $40 \mu \mathrm{g}$ of total cell extracts were prepared and run on SDS-PAGE for western blot analysis with the anti-GFP (upper panel) and $\beta$-actin antibodies (lower panel). The same blot was also probed with the anti-HuR antibody (3A2) to detect endogenous HuR (second panel)

which is a commonly used indicator for muscle differentiation efficiency. ${ }^{23}$ As earlier shown, ${ }^{6}$ depleting HuR expression by $\sim 70 \%$ (Figure $3 a$ ) significantly reduced the efficiency of muscle fiber formation (panel 2 in Figure $3 b$ and $c$ ). The rescue experiment, however, showed that unlike AP-HuR, the AP-HuRD226A mutant failed to rescue myogenesis in siRNA-HuR-treated myoblasts (Figure $3 b-d$ ). Therefore, these observations clearly show that an efficient cleavage of HuR by caspases is required for HuR to promote myogenesis.

\section{Association between HuR-CP1 and TRN2 prevents the} nuclear import of HuR in muscle cells. Recently, we showed that in HeLa cells undergoing apoptosis, the cleavage of HuR occurs only in the cytoplasm. ${ }^{11}$ We tested whether this is also the case during myogenesis, and showed that HuR-CP1 is generated only in the cytoplasmic fraction of differentiating muscle cells (Figure 4a). We also observed that HuR-CP1 levels become maximal in the cytoplasmic fraction at a time when HuR levels in the cytoplasm becomes clearly visible (Figure 4, compare lanes 5, 6, 7 with lane 8). In addition, blocking caspase activity with $10 \mu \mathrm{M}$ zVAD for $16 \mathrm{~h}$ at day 2 of differentiation of $\mathrm{C} 2 \mathrm{C} 12$ cells resulted in the nuclear sequestration of HuR (Figure $4 b$ ). The implication of a

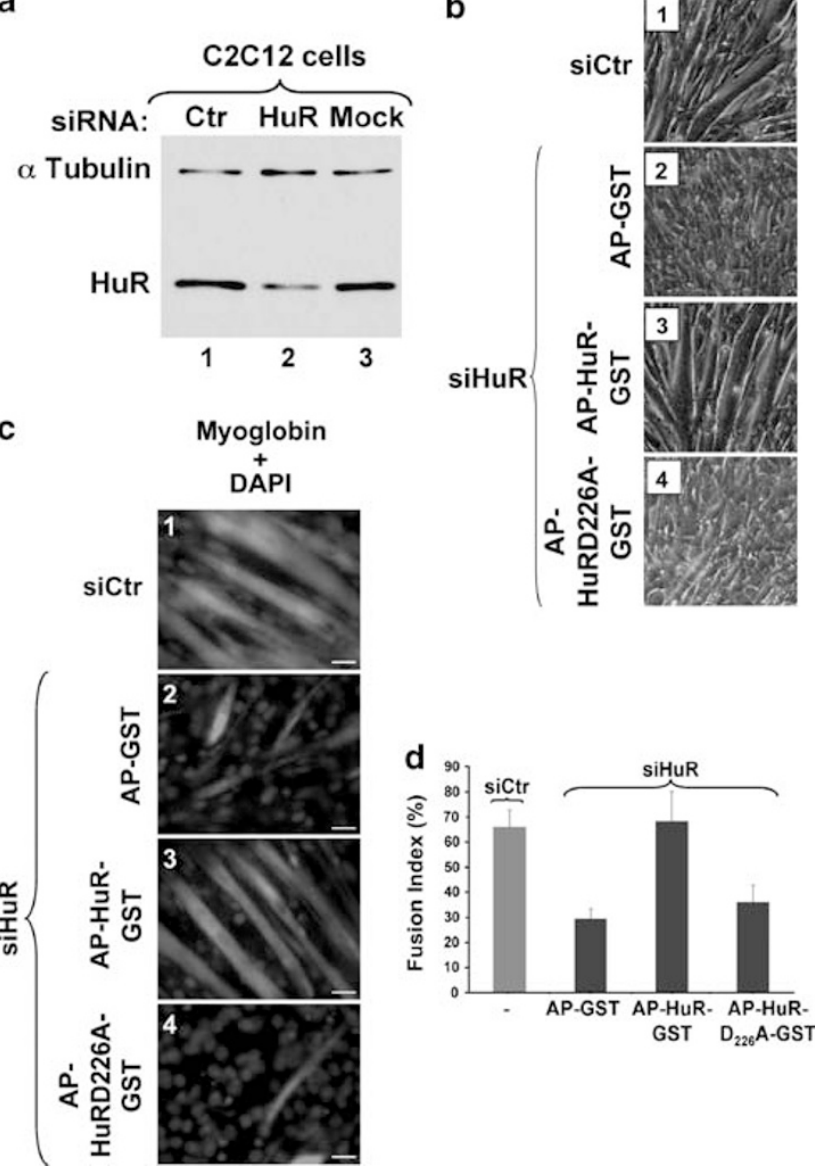

Figure 3 The cleavage of HuR at D226 residue is required for its ability to promote myogenesis. (a) $\mathrm{C} 2 \mathrm{C} 12$ cells treated with siRNA(si)-HuR or si-control (Ctr) as described ${ }^{6}$ were induced to differentiate; $5 \mu \mathrm{g}$ of total cell extract of cells on day 0 of differentiation was used for western blot analysis with the anti-HuR and anti- $\alpha$ tubulin (loading control) antibodies. Representative western blots of three independent experiments are shown. (b) AP-conjugated-GST, AP-HuR-GST, or AP-HuRD226A-GST were added twice to siHuR- or siCtr-treated $\mathrm{C} 2 \mathrm{C} 12$ cells (transfected $24 \mathrm{~h}$ before differentiation initiation and at the induction of differentiation). AP-GST and AP-HuR-GST were used, respectively, as negative and positive controls. Phase-contrast pictures of a single representative field of view for each cell treatment on day 3 of differentiation are shown. (c) siHuR- or siCtrtreated $\mathrm{C} 2 \mathrm{C} 12$ cells in the presence of $50 \mathrm{nM}$ AP-GST, AP-HuR-GST, or APHuRD226A-GST were fixed at day 3 of differentiation and immunofluorescence staining was performed against myoglobin and using DAPI. A single representative field for each cell treatment is shown. Bars, $20 \mu \mathrm{m}$. (d) The fusion index (percent differentiation) was determined by dividing the number of nuclei in myoglobinpositive myotubes by the total number of nuclei in a given microscopic field. For each experiment at least five different fields were counted. Error bars represent the S.E.M. of two independent experiments

caspases in the cytoplasmic accumulation of HuR in differentiated muscle fibers was also confirmed using our animal model. Indeed, we performed nuclear and cytoplasmic fractionation experiments using differentiated EDL muscle fibers that were isolated from caspase- $3+/+$ and $-1-$ mice. We observed that in the EDL muscles from caspase-3 -/- mice, the cytoplasmic levels of HuR were significantly lower than those from caspase- $3+/+$ mice (Figure 4c-e). Of note, the low levels of total extracts used from each fraction did not allow the detection of HuR-CP1 or 
a
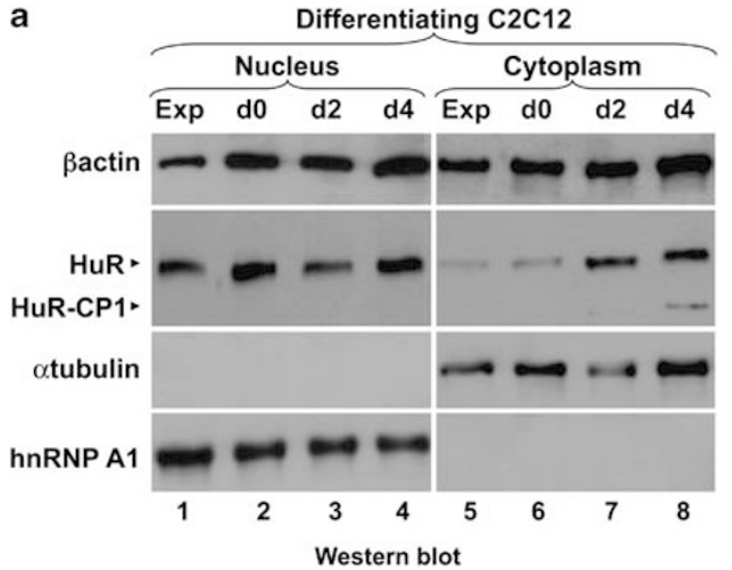

b

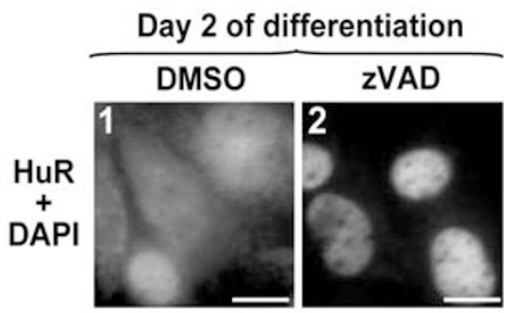

C

EDL muscle fiber from mice
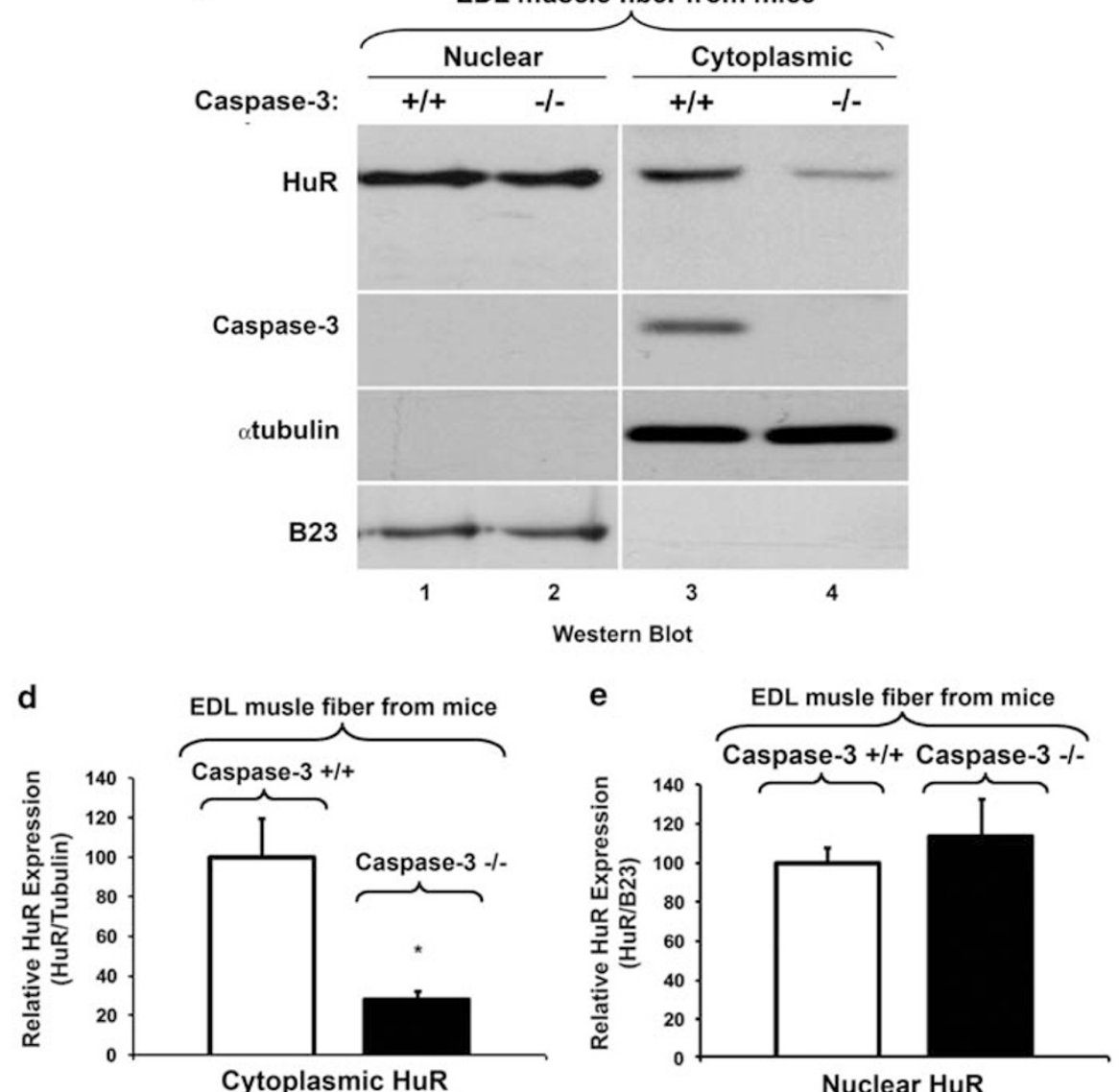

Nuclear HuR

Figure 4 The caspase-3-dependent cleavage of HuR occurs in the cytoplasm of muscle fibers both in differentiated C2C12 cells and in mice. (a) Nuclear/cytoplasmic fractionation was performed during $\mathrm{C} 2 \mathrm{C} 12$ differentiation, and western blotting analysis was performed on $35 \mu \mathrm{g}$ of cell extract for each time point. Membranes were probed with antibodies against HuR, $\beta$-actin, hnRNP A1 (a nuclear marker), and $\alpha$-tubulin (a cytoplasmic marker). Representative western blots of two independent experiments are shown. The white lines indicate intervening lanes that have been removed. (b) $\mathrm{C} 2 \mathrm{C} 12$ cells treated with DMSO or ZVAD on induction of differentiation were fixed at day 2, and analyzed by immunofluorescence staining for DAPI and against HuR. Representative images from two independent experiments are shown. Bars, $20 \mu \mathrm{m}$. (c) Representative western blot of the subcellular distribution of HuR in control $(+/+)$ and caspase-3-deficient $(-/-)$ mice. Nuclear and cytoplasmic extracts were obtained from EDL muscle. Western blot analysis was performed using $30 \mu \mathrm{g}$ of both nuclear and cytoplasmic extracts. As shown, blots were probed for HuR, caspase-3, the nuclear marker B23 (nucleophosmin), and the cytoplasmic marker $\alpha$-tubulin. (d, e) Quantification of the levels of HuR in the cytoplasmic (d) and nuclear (e) fractions from EDL muscles. Means $(+/-$ S.E. $)$ are shown and expressed as percent of control value. Asterisk denotes a significant difference $(P<0.05 ; n=4)$

caspase-3-CP in the cytoplamic fraction of EDL muscles from caspase- $3+/+$ mice. Together, these observations suggest that caspase activity is involved in the cytoplasmic accumulation of HuR during myogenesis.
Earlier experiments have shown that the amount of fulllength HuR that associates with TRN2 during myogenesis is around $10 \%$ of total HuR. ${ }^{10}$ This corresponds to the amount of HuR-CP1 generated in differentiating C2C12 cells $(\sim 11 \%)$. 
Moreover, the two amino-acid residues (Pro 222 and Met 223) of $\mathrm{HuR}$ that are required for HuR binding to $\mathrm{TRN}^{24}$ are located in the C-terminal region of HuR-CP1 (Figure 2a; Supplementary Figure S4a). These observations, combined with the fact that the cleavage of HuR during myogenesis occurs at the same time as and when the HuR/TRN2 complex is disrupted (between days 3 and 4$),{ }^{10}$ raised the possibility that by cleaving a portion of HuR, muscle cells generate HuR-CP1 to block the TRN2-mediated import of HuR. To test this possibility, we performed immunoprecipitation experiments using anti-TRN2 antibody on total extracts of differentiating $\mathrm{C} 2 \mathrm{C} 12$ cells that were earlier transfected with the GFP-HuR, -HuR-CP1, or -HuR-CP2 isoforms (Supplementary Figure S5). Western blot analysis using the anti-HuR antibody (3A2) showed that HuR and HuR-CP1 interact with TRN2 (Figure $5 \mathrm{a}$ ). As the epitope recognized by the 3A2 antibody is located in the first RNA recognition motif (RRM) of HuR, ${ }^{19}$ HuR-CP2 could not be detected using 3A2 (Figure 5a, lower panel), and, therefore, the same blots were then probed with the anti-GFP antibody. Although the association between HuR and HuR-CP1 with TRN2 was confirmed (Figure 5b, middle panels), we did not detect any interaction between HuR-CP2 and TRN2 (Figure 5b, compare bottom panel with top panel). The fact that both HuR and HuR-CP 1 associated with TRN2 to the same extent is a good indication that this $\mathrm{CP}$ has the ability to disrupt the HuR/TRN2 complex. To test this possibility, we performed an in vitro competition assay in which we disrupted the association between gluthation S-transferase (GST)-TRN2 and recombinant (Rec)-HuR using Rec-HuR-CP1 or Rec-HuR-CP2. Recombinant proteins had their GST tags removed using the PreScission Protease (GE Healthcare, Quebec, Canada) (Supplementary Figure S6). The association of Rec-HuR to GST-TRN2 beads as well as the disruption of this complex by Rec-HuR-CP1 or -HuR-CP2 was determined in vitro using western blot analysis with the anti-HuR monoclonal antibody. As expected, Rec-HuR associated to the GST-TRN2 beads, but not to empty GST beads (Figure 5c, compare lane 2 with lane 4). Our competition experiment, however, has shown that an increasing concentration of Rec-HuR-CP1, but not Rec-HuR-CP2, was able to compete away the association of HuR with GST-TRN2 (Figure 5d, compare lanes 1-5 with lanes 6with 10). These observations clearly show that HuRCP1 not only associates with TRN2 both in vitro and inside muscle cells, but also has the ability to disrupt the HuR/TRN2 complex.

The observations described above lead us to hypothesize that this CP is also able to disrupt the HuR/TRN2 complex in differentiating muscle cells, which would explain how HuR accumulates in the cytoplasm during muscle fiber formation. Several observations from different groups have shown that at early stages of the differentiation process (exponential and day 0 ), HuR is mainly localized in the nucleus. $5,6,10$ Therefore, if our hypothesis is true, the overexpression of HuR-CP1 in muscle cells at day 0 of myogenesis should result in the cytoplasmic accumulation of HuR. To test this hypothesis, we performed fluorescence experiments in which we followed the cellular localization of GFP-HuR in C2C12 cells at day 0 of differentiation in the presence or absence of AP-HuR-CP1GST or AP-HuR-CP2-GST. As expected, GFP-HuR, in the presence of AP-GST, localized mainly in the nucleus (Figure 5e, panels 1 and 2). However, there was a significant increase in the cytoplasmic level of GFP-HuR in cells treated with AP-HuR-CP1, but not in those treated with AP-HuR-CP2 (Figure 5e, compare panels 3 and 4 to 5 and 6). Together, these results suggest that by associating with TRN2, HuRCP1 disrupts the HuR/TRN2 complex leading to the cytoplasmic accumulation of HuR, which was earlier shown to be crucial in the promyogenic function of HuR. ${ }^{5,6,10}$ The fact that the overexpression of TRN2 in differentiating myoblasts significantly reduced the efficiency of myogenesis (Figure 6) strongly suggests that an increase in the nuclear import of HuR, as a result of an artificial elevation of TRN2 level, negatively affects muscle fiber formation.

It is well established that HuR promotes muscle fiber formation by stabilizing, in the cytoplasm, ARE-containing messages such as myogenin. ${ }^{5,10,25}$ HuR exercises this stabilization through its ability to specifically recognize the AREs in the 3'UTR of its target messages. ${ }^{5,10,25}$ Therefore, to determine whether the cleavage of HuR affects the fate of its mRNA targets, we assessed the association between HuRCP1 and HuR-CP2 and the HuR-binding site located in the 3'UTR of myogenin mRNA (myogenin ARE). Gel shift experiments using the different GST-tagged HuR isoforms showed that only HuR-CP1, but not HuR-CP2, associated with this ARE to the same extent as full-length HuR (Supplementary Figure S7). These data and the fact that HuR-CP1-induced cytoplasmic accumulation of HuR participates in myogenesis (Figures 5 and 6) raise the possibility that overexpressing HuR-CP1 in differentiating muscle cells could correlate with an increase in the stabilization of the myogenin mRNA. To test this possibility, we performed an actinomycin $D$ (ActD) pulse-chase experiment ${ }^{26}$ on differentiating $\mathrm{C} 2 \mathrm{C} 12$ cells treated with AP-HuR or AP-HuR-CP1. We observed that an excess of AP-HuR or AP-HuR-CP1 in C2C12 cells triggers an increase in the half-life of the myogenin mRNA, albeit to a different extent (Figure $7 \mathrm{a}$ and $\mathrm{b}$ ). These observations strongly indicate that HuR-CP1, by blocking the nuclear import of endogenous HuR (Figure $5 \mathrm{~d}$ ), indirectly increases the half-life of the myogenin mRNA. However, it is also possible that HuR-CP1, through its RRMs 1 and 2, is able to independently promote myogenesis by directly stabilizing the myogenin mRNA. To investigate which of these two possibilities is true, we assessed whether HuR-CP1 is able to rescue the expression of myogenin mRNA in HuR knockdown $\mathrm{C} 2 \mathrm{C} 12$ cells. We depleted HuR expression with high efficiency $(>85 \%)$ using siRNA-HuR ${ }^{10}$ in undifferentiated myoblasts (Figure 7c). These cells were then induced to differentiate in the presence of $50 \mathrm{nM}$ AP-GST, AP-HuR, or AP-HuR-CP1. As expected, ${ }^{6}$ in the absence of $\mathrm{HuR}, \mathrm{C} 2 \mathrm{C} 12$ cells failed to express the myogenin mRNA (Figure 7d, compare lanes 1 and 2 with lane 3 ). However, the expression of this mRNA was rescued by only AP-HuR, but not AP-HuR-CP1 (Figure 7d, compare lanes 6 and 9). Likewise, only AP-HuR, but not AP-HuR-CP1, was able to rescue myogenesis in $\mathrm{C} 2 \mathrm{C} 12$ cells depleted of HuR (Figure 7e and f). Together, these data strongly suggest that the stabilization effect of HuR-CP1 on the myogenin mRNA is an indirect event, which occurs only through its ability to promote the cytoplasmic localization of HuR. 


\section{Discussion}

Our investigations have uncovered one of the mechanisms by which posttranscriptional regulatory events promote muscle fiber formation. We show that during myogenesis in cultured muscle cells or during the regeneration of skeletal muscle tissues in mice, the RNA-binding protein HuR undergoes a caspase-mediated cleavage and this proteolytic event is required for myotube formation. The cleavage of HuR generates HuR-CP1, a $24 \mathrm{kDa}$ fragment that, by associating with the HuR import factor TRN2, promotes the cytoplasmic accumulation of HuR and triggers an increase in the half-life of the HuR-mRNA target myogenin. Therefore, we suggest a model whereby during myotube formation, a percentage of HuR is cleaved, generating HuR-CP1 that retains the ability to associate with TRN2. Through this association, HuR-CP1 sequesters TRN2 leading to the cytoplasmic accumulation of HuR. In the cytoplasm, HuR stabilizes several mRNA targets such as myogenin, thereby participating in the events that lead to myotube formation (Figure 8).

Our earlier observations have shown that the cleavage of HuR is required to promote apoptosis in cells exposed to lethal stresses. ${ }^{11}$ In this study, we show that cleavage is also an important regulator of the promyogenic function of HuR. It was intriguing to note that a similar regulatory mechanism is used to mediate two opposite functions of HuR: the induction of cell death and the promotion of cell differentiation. Our results, however, are consistent with the earlier reports implicating effector caspases such as caspases-3 and -9 in both stressinduced cell death and the promotion of muscle cell differentiation. ${ }^{12,15,27}$ Here, we show that HuR cleavage is triggered only during the fusion step of myoblasts leading to myotube formation (Figure 1a and b). Interestingly, this is also the case for the activation of both caspases- 3 and -9 , which occurs only during the fusion step of muscle cells ${ }^{15}$ (Figure 1a). Whereas the function of caspases in the differentiation process for a number of cell types is shown, ${ }^{28}$ the regulatory mechanisms involved and their substrates remain largely unknown. The fact that caspase-3 has an important function in HuR proteolysis during apoptosis, ${ }^{11}$ and our data showing that the cleavage of HuR is significantly reduced in both cardiotoxin-treated TA muscle from caspase$3-/-$ mice, as well as in myotubes exposed to zVAD (Figure 1), indicates that HuR could be one of the main substrates of caspase-3 during muscle cell differentiation.
Although this is likely to be the case, more studies are needed before a definitive conclusion can be made.

Our data show that although only $10-15 \%$ of total HuR is cleaved during the myogenic process both in $\mathrm{C} 2 \mathrm{C} 12$ muscle cells and in mice (Figure 1), this event is required for the promyogenic activity of HuR (Figure 3). Indeed, here we show that mutating the D226 residue to A generates an HuR isoform that is not only protected from caspase-induced cleavage ( $<1 \%$ of HuR-CP1 produced) (Figure $2 \mathrm{c}$ ), but also failed to reestablish myogenesis in siRNA-HuR-treated myoblasts (Figure 3). This indicates that the fraction of cleaved HuR $(10-15 \%)$ is crucial for the efficient execution of the myogenic process. Moreover, our data indicate that $\sim 90 \%$ of total HuR is not cleaved and that a portion of this uncleaved HuR accumulates in the cytoplasm during myogenesis (Figure 4). ${ }^{10}$ Therefore, these results, and the fact that an excess of HuR-CP1 correlates with the stabilization of myogenin mRNA (Figure $7 a$ and $b$ ), argue that both the cleaved and the noncleaved isoforms of HuR collaborate together to ensure proper myogenesis.

As a function of caspases and other proapoptotic factors in myogenesis has already been suggested, ${ }^{12,15}$ we were not surprised to observe that HuR is cleaved in a caspasedependent manner in this process. It has been shown that caspases-3 and -9, which are important effectors of apopto$\mathrm{sis}^{27}$ have a major function in myogenesis. ${ }^{12,15}$ Establishing a link between posttranscriptional regulators such as HuR, and important players in cell death mechanisms such as caspases will increase our understanding of how the skeletal muscle tissues can deal with different extracellular assaults to maintain their integrity. Furthermore, by continuing to investigate the functions of the CPs of HuR, and the regulation of their generation, we will gain a greater understanding of how important posttranscriptional regulators such as HuR can be involved in promoting different vital cellular processes.

\begin{abstract}
Materials and Methods
Plasmid construction and protein purification. AP-HuR-CP1-GST and AP-HuR-CP2-GST were generated by cloning HuR-CP1 and HuR-CP2 fragments into the AP/GST vector as described. ${ }^{6}$ The PCR amplification of HuR$\mathrm{CP} 1$ and HuR-CP2 was performed using the GST-HuR plasmid as template ${ }^{29}$ with the primers HuR-CP1-GST-forward (5'- ggc gcg gcc gca tct aat ggt tat gaa gac cac $\left.-3^{\prime}\right)$ and AP-HuR-CP1-reverse ( $5^{\prime}$ - ggc ctc gaggtgatc gac gcc cat ggg $\left.-3^{\prime}\right)$, and HuR-CP2-GST-forward (5'- ggc gcg gcc gca cac atg agc ggg ctc tct $\left.-3^{\prime}\right)$ and
\end{abstract}

Figure 5 HuR-CP1 associates with TRN2, disrupts in vitro the HuR-TRN2 complex and interferes with the nuclear import of HuR in undifferentiated C2C12 cells. (a, b) Total extracts from transfected C2C12 cells expressing GFP-HuR, GFP-HuR-CP1, GFP-HuR-CP2, or GFP-HuRD226A were used for immunoprecipitation with anti-TRN2 antibody or IgG control. The immunoprecipitate $(P)$ and the supernatant $(S)$ were analyzed by western blot using antibodies against HuR (a) and GFP (b). (a) End-HuR is the endogenous HuR found in the cells. (a, b) The position of GFP, GFP-HuR, GFP-HuR-CP1, GFP-HuR-CP2, or GFP-HuRD226A proteins are indicated. (c) GST-TRN2 beads were prepared as described in the Material and methods section. A total of $0.225 \mathrm{nmol}$ Rec-HuR was incubated with either beads alone (lanes 1 and 2) or with $0.15 \mathrm{nmol}$ GSTTRN2 beads (lanes 3 and 4). The flow-through (FT) represents the soluble fraction without the beads and the pellet $(P)$ represents the pellet that contains the beads. FT and P fraction were used for western blot with the anti-HuR antibody. (d) The P fraction from C was incubated sequentially for 30 min with increasing concentration of Rec-HuR-CP1 or Rec-HuR-CP2 $(0.0225,0.045,0.225,0.45$, and $1.125 \mathrm{nmol})$ and the FT of each treatment was collected and used for western blot with the anti-HuR antibody. To avoid the high background that was generated by the increasing amount of Rec-HuR-CP1 when revealing the western blot by ECL, we separated the membrane into two. The upper part was used to reveal the eluted Rec-HuR and the lower part was used to detect the added Rec-HuR-CP1. The membrane revealing the Rec-HuR was exposed for a longer time period than the exposure time used to reveal the Rec-HuR-CP1. Representative western blots of two independent experiments are shown. (e) C2C12 myoblasts were transfected with GFP-HuR and $48 \mathrm{~h}$ later when the cells were confluent as they were induced for differentiation. Two hours later, these cells were treated with AP-GST, AP-HuR-CP1-GST, or AP-HuR-CP2-GST. Eight hours later, the cells were fixed and stained for DAPI. A single representative field for each cell treatment from three independent experiments is shown. Bars, $20 \mu \mathrm{m}$ 
AP-HuR-CP2-reverse ( $5^{\prime}$ - ggc ctc gag ttt gtg gga ctt gtt ggt ttt $\left.-3^{\prime}\right)$, respectively. The Not1/Xho1 fragments of HuR-CP1 and -CP2 PCR products were inserted into the Not1/Xho1 sites of the Gateway AP-GST vector to produce AP-HuR-CP1-GST and AP-HuR-CP2-GST. AP-HuR-GST and AP-HuRD226A-GST were generated and used as earlier described. ${ }^{11}$

The AP-tagged proteins were expressed and produced as described earlier ${ }^{6}$ except for the following modifications: the proteins were eluted in a buffer containing $300 \mathrm{mM}$ Tris pH 8.8 and $120 \mathrm{mM} \mathrm{NaCl}$, with $10 \mathrm{mM}$ glutathione for the first elution and $20 \mathrm{mM}$ gluthatione for further elutions.
The GFP-tagged HuR mutants, GFP-HuR, GFP-HuRD226A, GFP-HuRD254A, and GFP-HuRD256A were generated and used as described in Mazroui et al..$^{11}$ GFP-HuRCP1 and GFP-HuR-CP2 were generated as follows. DNA coding for the first 226 amino acids of HuR (1-226) (HuR-CP1) or the last 100 amino-acids (227-326) (HuR-CP2) was amplified by PCR, using wild-type HuR cDNA as the template. A Bglll site was created at the $3^{\prime}$-end and an EcoRl was generated at the $5^{\prime}$-end of the PCR product for both HuR-CP1 and HuR-CP2. GFP-HuR-CP1 was generated using the following primers forward: $5^{\prime}$-GGC AGA TCT AAT GGT TAT GAA GAC CAC A-3' and reverse: 5'-GGC GAA TTC TTA ATC GAC GCC CAT GGG-3'. We generated GFP-HuR-CP2 a

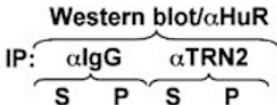

End-HuR

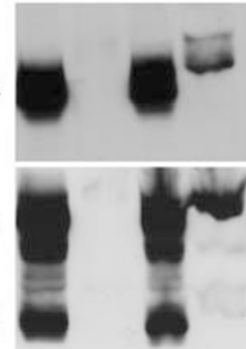

End-HuR

GFP-HuR-CP1

End-HuR

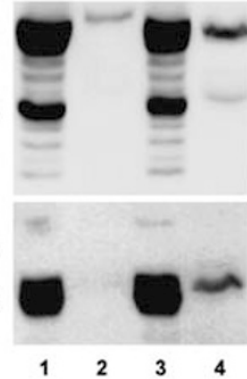

C

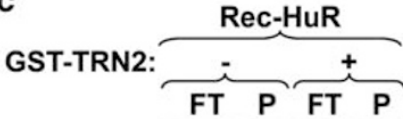

beads

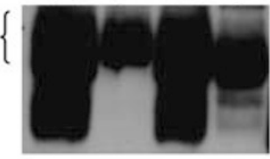

\begin{tabular}{llll}
1 & 2 & 3 & 4 \\
\hline
\end{tabular}

d Competing

proteins: Rec-HuR-CP1
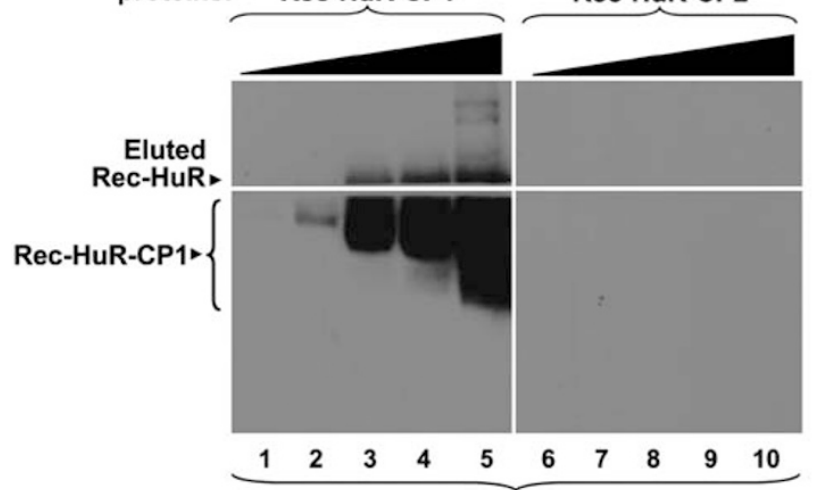

Western blot using $\alpha$.HuR b

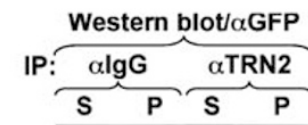

GFP

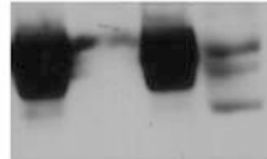

GFP-HuR

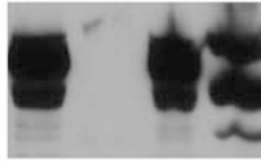

GFP-HuR-CP1

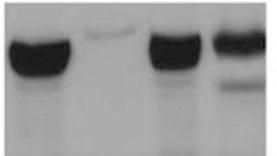

GFP-HuR-CP2

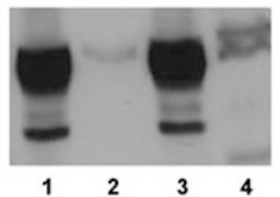

e

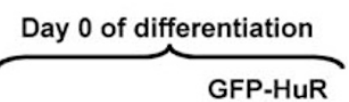

$\triangleleft$ Rec-HuR

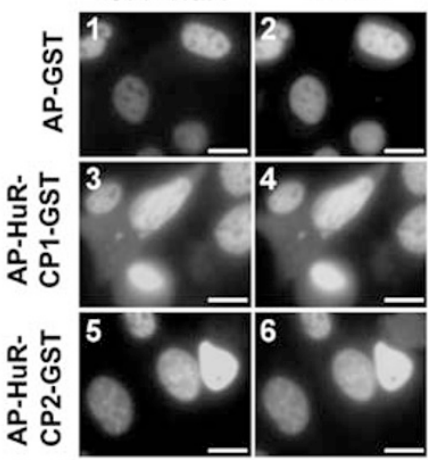


a Day 3 post differentiation induction
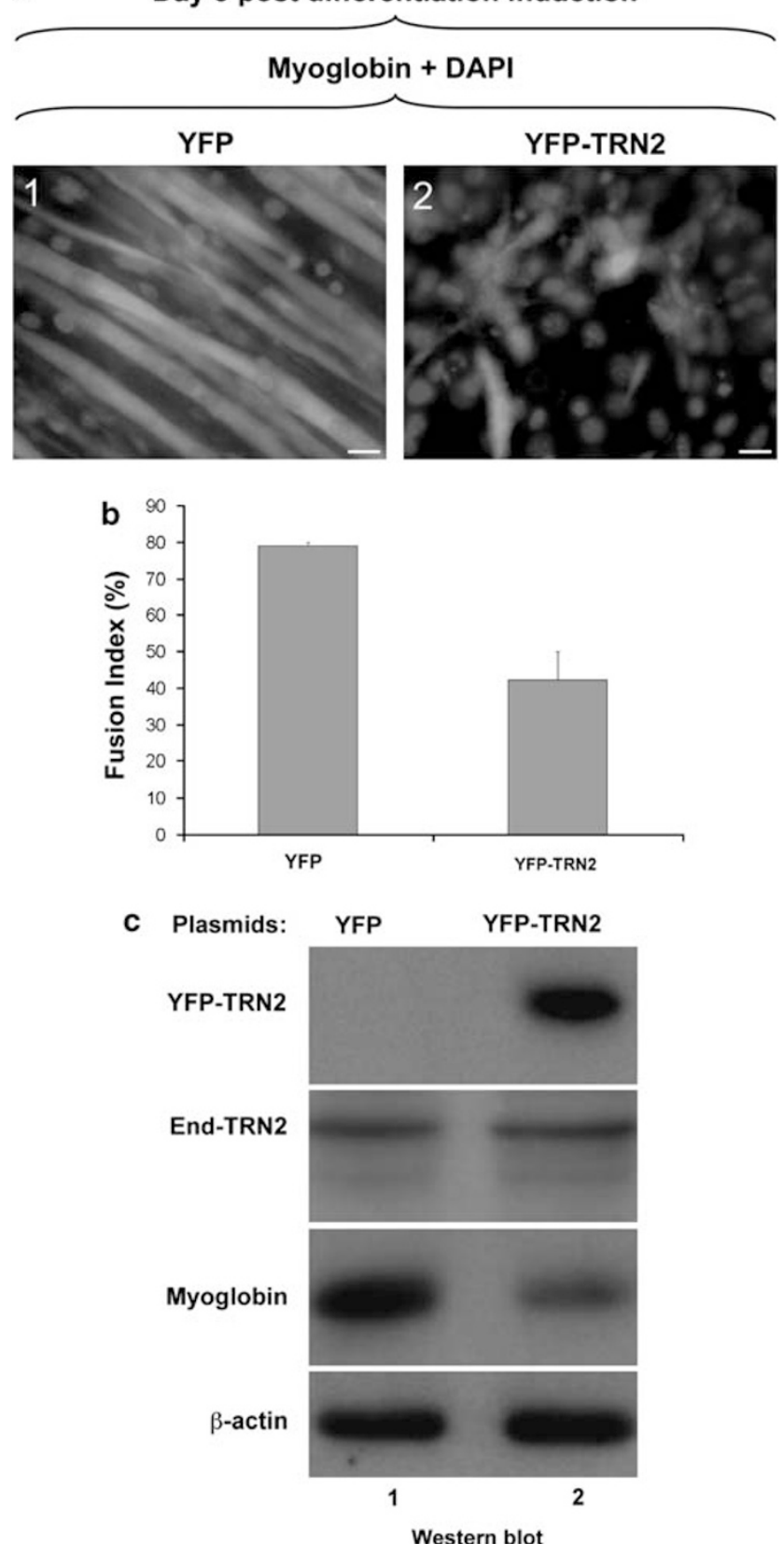

Figure 6 The overexpression of TRN2 in myoblasts significantly reduces their myogenic potential. (a) $\mathrm{C} 2 \mathrm{C} 12$ myoblasts were transfected twice with YFP or YFPTRN2, at $50 \%$ and again the following day at $75 \%$ confluency. Cells were fixed at day 2 of differentiation and DAPI as well as immunofluorescence staining was performed using anti-myoglobin antibody. A single representative field for each cell treatment is shown. Bars, $20 \mu \mathrm{m}$. (b) The fusion index is shown and was calculated as described above. Error bars represent the S.E.M. of two independent experiments. (c) Total cell extracts from differentiated YFP or YFP-transfected $\mathrm{C} 2 \mathrm{C} 12$ cells were used for western blot analysis with anti-YFP, -TRN2, myoglobin, and $\beta$-actin antibodies. Representative western blots of two independent experiments are shown

using forward: 5'-GGC AGA TCT CAC ATG AGC GGG CTC TCT-3' and reverse: $5^{\prime}$-GGC GAA TTC TTA GTA AGC TGC GAG AGG AG-3'. The PCR fragments were cloned into the Bglll/EcoRI sites of pAcGFP1-C1 vector (BD Bioscience, San Jose, CA, USA) and both fragments inserted in frame with GFP CDNA.
GST-HuR, GST-HuR-CP1, and GST-HuR-CP2 were generated as follows. Fulllength $\mathrm{HuR}$ or the regions representing its cleavage fragments were amplified by PCR using the GFP-HuR, GFP-HuR-CP1, or GFP-HuR-CP2 plasmids described above. An EcoRI site was created at the $5^{\prime}$-end and a Notl site was generated at the $5^{\prime}$-end of the PCR products. GST-HuR was generated using the following primers EcoRI-HuR-F: $5^{\prime}$-CCG GAA TTC CGG TCT AAT GGT TAT GAA GAC CAC- $3^{\prime}$ and Notl-HuR-R: $5^{\prime}$-GGC GCG GCC GCG CCT TAT TTG TGG GAC TTG TTG GT-3'; GST-HuR-CP1 was generated using EcoRI-HuR-F primer and Notl-HCP1-R: $5^{\prime}-$ GGC GCG GCC GCG CCA TCG ACG CCC ATG GGG GA-3'; and GST-HuR-CP2 was generated using EcoRI-HCP2-F: 5'-CCG GAA TTC CGG CAC ATG AGC GGG CTC TCT- $3^{\prime}$ and Notl-HuR-R primer. The PCR fragments were cloned into the EcoRI/Notl sites of pGEX-6P-1 vector (GE Healthcare) in frame with GST cDNA.

The GST-conjugated proteins were prepared as described. ${ }^{29} \mathrm{~A}$ prokaryotic vector expressing GST-TRN2 was transfected into competent BL21 bacteria. On IPTG induction, the bacteria were lysed and incubated for $4 \mathrm{~h}$ with glutathione sepharose beads (GE Healthcare). The GST-TRN2 beads were then washed at least four times and incubated overnight with $5 \mu \mathrm{g}$ of Rec-HuR. The GST-tag was removed from GST-HuR, GST-HuR-CP1, and GST-HuR-CP2 using PreScission Protease (GE Healthcare) according to the manufacturer's instructions.

YFP-TRN2 was generated by amplifying TRN2 from GST-TRN2 template by PCR, creating a BamHI and a Notl restriction site at its $3^{\prime}$ - and $5^{\prime}$-end, respectively. The PCR fragment was then cloned into the BamHI/Notl sites of YFP-pcDNA3 (generous donation from $\mathrm{J}$ Pelletier) in frame at the C-terminal end of the YFP CDNA. Primers used were $5^{\prime}$-CGC GGA TCC GCG GAC TGG CAG CCA GAC GAG-3' (forward) and $5^{\prime}$-GGC GCG GCC GCG CCC TAG ACC CCA TAG AAA GCC G-3' (reverse).

Cell culture and transfection, zVAD, and cell-permeable protein treatments. C2C12 cells (ATCC, Manassas, VA, USA) were grown, transfected with siRNAs and differentiation was induced as described earlier. ${ }^{6,10}$ To examine caspase-dependent cleavage of HuR by western blotting, ZVAD (Sigma-Aldrich, Montreal, QC, Canada) was added directly to $\mathrm{C} 2 \mathrm{C} 12$ myotubes on day 3 of differentiation for $16 \mathrm{~h}$, at a final concentration of $10 \mu \mathrm{M}$, after which the cells were supplemented with another $10 \mu \mathrm{M}$ zVAD for $12 \mathrm{~h}$ before harvesting on day 4 as described below. To assess the localization of HuR by immunofluorescence, zVAD was added on day 1 of differentiation for $16 \mathrm{~h}$, at a final concentration of $10 \mu \mathrm{M}$, and then supplemented with another $10 \mu \mathrm{M}$ zVAD for $12 \mathrm{~h}$ before the cells were fixed on day 2 .

The GFP-conjugated constructs were transfected into muscle cell as described. ${ }^{6,11}$ For cell-permeable fusion protein experiments, all proteins were generated as described above and added to $\mathrm{C} 2 \mathrm{C} 12$ myoblasts at a final concentration of $50 \mathrm{nM}$ both on the day before and the day of differentiation of induction as described. ${ }^{6}$

In vivo muscle regeneration. For regeneration studies, $10 \mu \mathrm{l}$ of cardiotoxin (Latoxan, Rosans, France) diluted at $10^{-5} \mathrm{M}$ was directly injected into the TA muscle of 4-week-old wild-type or caspase-3 -/ - C57BL/10 mice (obtained from Charles River Laboratories, St.-Constant, Canada), as described earlier; ${ }^{20,30} 1,3,7$, and 14 days later, cardiotoxin-injected and control-untreated TA muscles were rapidly excised and frozen in melting isopentane precooled with liquid nitrogen (for histological analysis), or directly frozen in liquid nitrogen (for western blots).

Histological analysis was performed on cross-sections of TA muscles that were stained with hematoxylin and eosin, dehydrated in a series of alcohol solutions, cleared with xylene, and mounted using permount (Fisher Scientific, Montreal, QC, Canada). For western blotting, total protein extracts were obtained from whole muscle samples homogenized in $300 \mathrm{ml}$ of RIPA buffer $(50 \mathrm{mM}$ Tris, $\mathrm{pH} 7.5$, $150 \mathrm{mM} \mathrm{NaCl}, 1 \%$ NP-40, 0.1\% SDS Sigma, Montreal, QC, Canada). Protein concentrations were determined using the Bradford Assay (Bio-Rad, Mississauga, ON, Canada). A total of $80 \mathrm{ig}$ of protein extract was resolved by SDS-PAGE and transferred onto polyvinylidene fluoride (PVDF) membranes (Millipore, Billerica, MA, USA). The blots were first stained with Memcode (Thermo Scientific, Nepean, ON, Canada) to ensure equal loading, subsequently probed with the $3 A 2$ antibody against HuR, ${ }^{19}$ or antibodies against caspase-3 (Cell Signaling, Boston, MA, USA) or myogenin (Developmental studies Hybridoma Bank, lowa, IA, USA) and revealed using ECL (Amersham, Montreal, QC, Canada).

Immunoblotting, immunofluorescence, subcellular fractionation, and preparation of cell extracts. Total cell extracts were prepared as described earlier. ${ }^{6}$ The nuclear and cytoplasmic fractions from differentiating $\mathrm{C} 2 \mathrm{C} 12$ cells were prepared as described. ${ }^{6,10}$ Western blotting was performed as described 
a
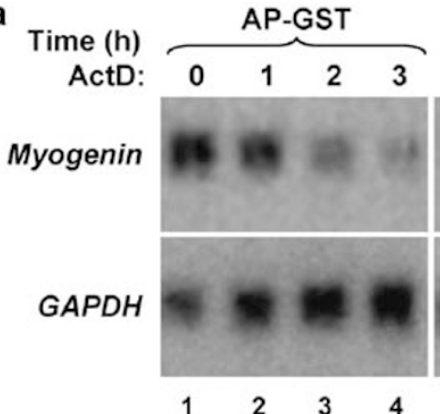

AP-HuR-GST

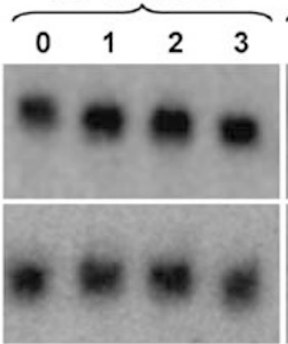

AP-HuR-CP1-GST

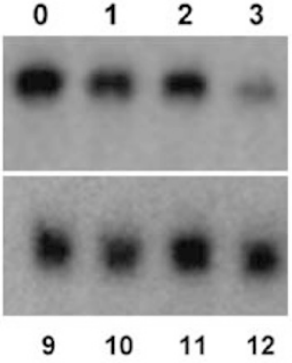

b

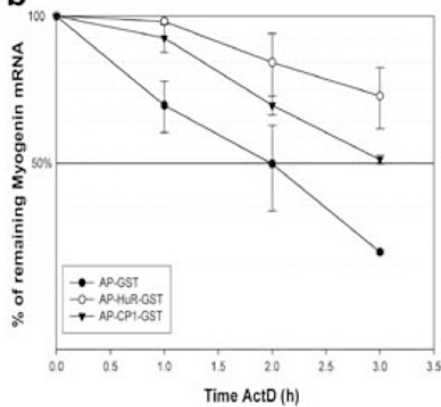

C

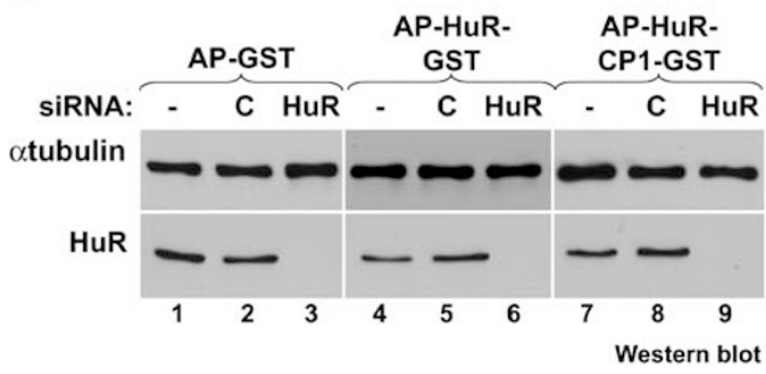

d
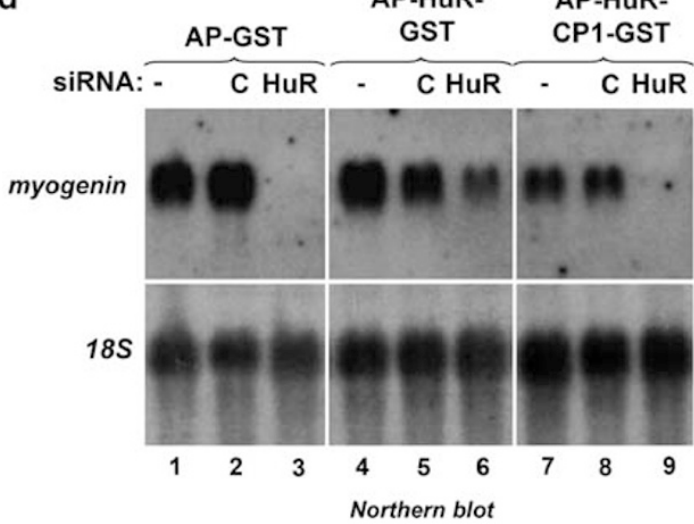

Northern blot e

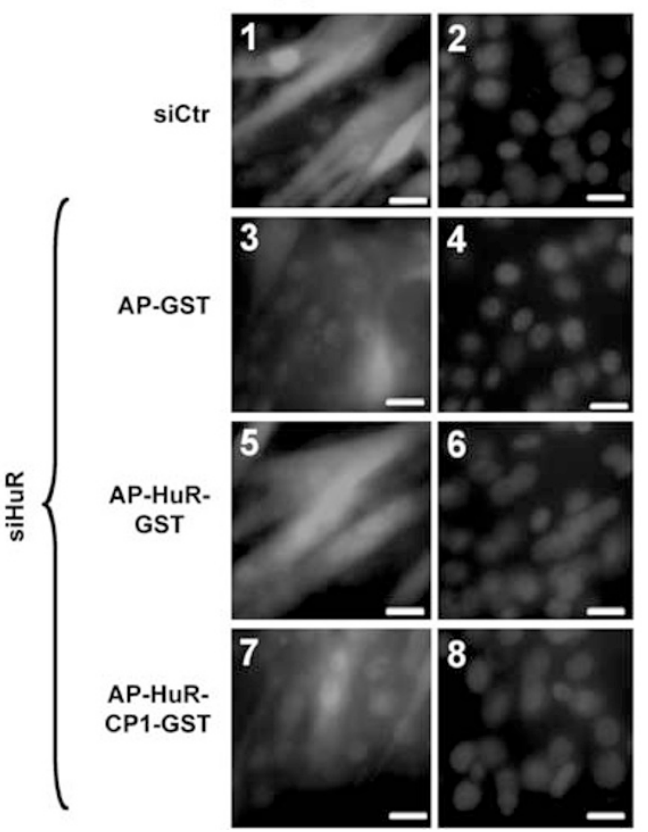

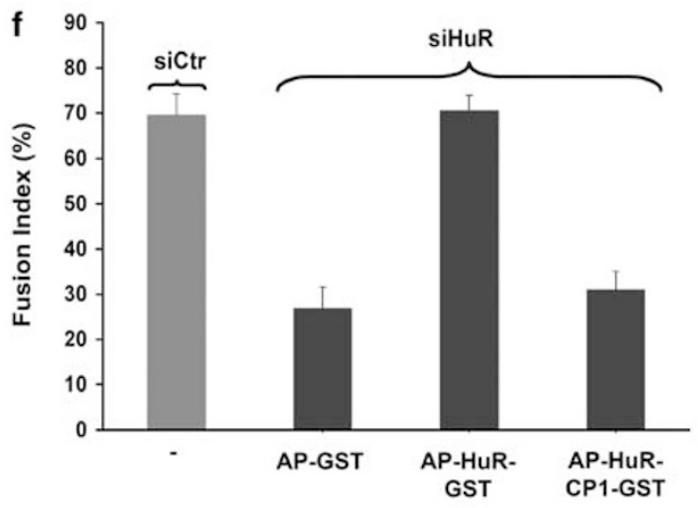

Figure 7 To promote the expression of myogenin mRNA and myogenesis, HuR-CP1 requires endogenous HuR. (a) Differentiation was induced in C2C12 cells, which were then treated, $24 \mathrm{~h}$ later, with AP-GST, AP-HuR-GST, or AP-HuR-CP1-GST. One hour after treatment with AP conjugates, cells were treated with ActD for $0,1,2$, or $3 \mathrm{~h}$. The mRNAs of myogenin and GAPDH (loading control) were detected using northern blot. (b) The stability of myogenin mRNA was quantified using the ImageQuant software. The percent remaining of myogenin mRNA was defined as follow: (1) by dividing the band intensities of myogenin over GAPDH at each time point, (2) each of these numbers was then normalized against the abundance of myogenin mRNA at $0 \mathrm{~h}$ of ActD treatment, considered as $100 \%$, and (3) the obtained percentages were plotted on a logarithmic scale. The half-life of the myogenin mRNA (50\%) was determined by calculating the remaining mRNA relative to the original abundance of message. Error bars represent the S.E.M. of two independent experiments. (c, d) AP-GST, -HuR-GST, or -HuR-CP1-GST were introduced to control (c) siRNA- or HuR-siRNA-treated C2C12 cells that were induced to differentiate for 2 days. Total extracts from these cells were prepared and then used for western blots with anti-HuR and - $\alpha$-tubulin antibodies (c) or northern blot analysis to assess the expression of myogenin mRNA and the $18 \mathrm{~S}$ (loading control) (d). (e) Knockdown of HuR expression by siRNAs duplexes, treatment with AP-GST, AP-HuR-GST, and AP-HuR-CP1 and the immunofluorescence experiments were performed as described in Figure 3c. A single representative field for each cell treatment is shown. Bars, $20 \mu \mathrm{m}$. (f) The fusion index was determined as in Figure 3d. Error bars represent the S.E.M. of two independent experiments 


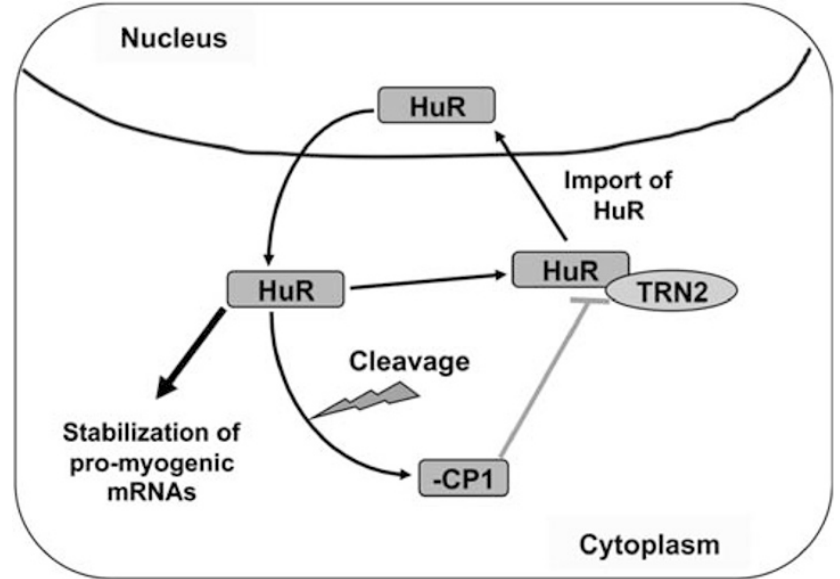

Figure 8 Model depicting how HuR cleavage participates in the promyogenic function of HuR. During the early steps of myogenesis, the nuclear import of HuR is ensured by the import factor TRN2. ${ }^{10}$ During fusion, in which myoblasts form myotubes, HuR is needed in the cytoplasm. At this stage, HuR is cleaved by caspases generating HuR-CP1, which in turn blocks the TRN2-mediated import of HuR. HuR-CP1 competes with HuR and forms a stable complex with TRN2. HuR then accumulates in the cytoplasm and stabilizes mRNA such as myogenin, thus promoting myogeneis ${ }^{5,6}$

earlier ${ }^{19}$ and blots were probed with antibodies against HuR (3A2), ${ }^{19}$ caspase-3 (Cell Signaling), myogenin (F5D), MyHC (MF-20) (Developmental studies Hybridoma Bank), $\alpha$-tubulin (Sigma-Aldrich), GFP (Clonetech, Mountain View, CA, USA), myoglobin (DAKO, Mississauga, ON, Canada), $\beta$-actin (Sigma), and hnRNP A1 (Abcam, Cambridge, MA, USA). Immunofluorescence was performed as described earlier ${ }^{6}$ using antibodies against HuR, myoglobin, and $\mathrm{MyHC}$ and staining with DAPI.

Cytoplasmic and nuclear extracts from whole EDL muscle samples were prepared using the ProteoJET cytoplasmic and nuclear protein extraction kit according to manufactures instructions (Fermentas Life Sciences, Burlington, ON, Canada). Samples were separated by SDS-PAGE and transferred to PVDF membranes. Western blots were performed using antibodies to $\operatorname{HuR}\left(3 \mathrm{~A} 2^{19}\right)$, caspase-3, $\alpha$-tubulin, and B23 (Sigma-Aldrich) and suitable HRP-conjugated secondary antibodies. Blots were exposed using western lightning enhanced luminescence reagent (PerkinElmer, Woodbridge, ON, Canada) and developed with CL-XPosure film (Thermo Scientific) and a Kodak X-OMAT 2000A processor. Blots were subsequently quantified through densitometry analysis (National Institutes of Health ImageJ).

Immunoprecipitation analysis. Total cell lysates for immunoprecipitation were prepared from $\mathrm{C} 2 \mathrm{C} 12$ cells that were transfected with GFP, GFP-HuR, GFPHuR-CP1, and GFP-HuR-CP2 DNA constructs before the initiation of differentiation. $\mathrm{C} 2 \mathrm{C} 12$ cells at day 2 of differentiation were scraped in phosphate-buffered saline and centrifuged at 2000 r.p.m. for $5 \mathrm{~min}$ at $4^{\circ} \mathrm{C}$. A measure of $2 \mathrm{mg}$ of total cell extracts prepared as described ${ }^{10}$ were incubated with protein $A$ beads (GE Healthcare) earlier bound to anti-TRN2 or IgG control (BioCan, Jackson ImmunoResearch Laboratories, West Grove, PA, USA) antibodies as described for $4 \mathrm{~h}$ at $4{ }^{\circ} \mathrm{C}$, and then washed with RIPA buffer. The immunoprecipitate was resuspended as described ${ }^{10}$ and then analyzed by western blot.

Gel shift assay. Gel shift experiments were performed as described earlier ${ }^{26}$ with the following modifications. As a probe, we used a ${ }^{32} \mathrm{P}$-UTP-labeled cRNA corresponding to the myogenin $3^{\prime}$ UTR ARE. ${ }^{6}$ The labeled CRNA probe was incubated as described earlier ${ }^{26}$ with $300 \mathrm{ng}$ of recombinant GST-HuR, GST-HuRCP1, and GST-HuR-CP2.

Northern (RNA) blot analysis and actinomycin D pulse-chase experiments. Northern blot analysis was performed using $12 \mu \mathrm{g}$ of total RNA as described earlier ${ }^{10}$ with the following modifications. Briefly, after transferring the RNA onto a hybond-N membrane (GE Healthcare), the RNA was UV-cross-linked and the blots were hybridized with myogenin and GAPDH cDNA probes. ${ }^{6}$ The stability of myogenin mRNA was assessed by treating $\mathrm{C} 2 \mathrm{C} 12$ cells, after $16 \mathrm{~h}$ of differentiation, with $50 \mathrm{nM}$ of AP-GST, AP-HuR-GST, or AP-HuR-CP1-GST; $1 \mathrm{~h}$ later, cells were treated with the RNA pol II inhibitor ActD (Sigma-Aldrich) at a concentration of $5 \mu \mathrm{g} / \mathrm{ml}$. Total RNA was isolated from the cells after $0,1,2$, and $3 \mathrm{~h}$ after ActD treatment using TRIzol reagent (Invitrogen, Carlsbad, CA, USA), and analyzed by northern blotting.

\section{Conflict of interest}

The authors declare no conflict of interest.

Acknowledgements. We thank Drs S Di Marco and V Dormoy-Raclet for their helpful suggestions throughout the progress of this work. We also thank Ms C Clow for her technical help with the in vivo experiments, and Mr N Punjani for his assistance with the production of cleavable GST-plasmids and proteins. This work was supported by a Cole Foundation fellowship for PB, a FRSQ Master's fellowship for CN, a CIHR Doctoral Research Award for CvR, and a CIHR (MOP-89798) operating grant to IG. IG is a recipient of a Tier II Canada Research Chair.

1. Charge SB, Rudnicki MA. Cellular and molecular regulation of muscle regeneration Physiol Rev 2004; 84: 209-238.

2. Kaeser MD, Emerson BM. Remodeling plans for cellular specialization: unique styles for every room. Curr Opin Genet Dev 2006; 16: 508-512.

3. Sabourin LA, Rudnicki MA. The molecular regulation of myogenesis. Clin Genet 2000; 57 : 16-25.

4. Lassar AB, Skapek SX, Novitch B. Regulatory mechanisms that coordinate skeletal muscle differentiation and cell cycle withdrawal. Curr Opin Cell Biol 1994; 6: 788-794.

5. Figueroa A, Cuadrado A, Fan J, Atasoy U, Muscat GE, Munoz-Canoves $P$ et al. Role of HuR in skeletal myogenesis through coordinate regulation of muscle differentiation genes. Mol Cell Biol 2003; 23: 4991-5004.

6. van der Giessen K, Di-Marco S, Clair E, Gallouzi IE. RNAi-mediated HuR depletion leads to the inhibition of muscle cell differentiation. J Biol Chem 2003; 278: 47119-47128.

7. von Roretz C, Gallouzi IE. Decoding ARE-mediated decay: is microRNA part of the equation? J Cell Biol 2008; 181: 189-194.

8. Barreau C, Paillard L, Osborne HB. AU-rich elements and associated factors: are there unifying principles? Nucleic Acids Res 2005; 33: 7138-7150.

9. Deschenes-Furry J, Belanger G, Mwanjewe J, Lunde JA, Parks RJ, Perrone-Bizzozero N et al. The RNA-binding protein HuR binds to acetylcholinesterase transcripts and regulates their expression in differentiating skeletal muscle cells. J Biol Chem 2005; 280 25361-25368.

10. van der Giessen K, Gallouzi IE. Involvement of transportin 2-mediated HuR import in muscle cell differentiation. Mol Biol Cell 2007; 18: 2619-2629.

11. Mazroui R, Di Marco S, Clair E, von Roretz C, Tenenbaum SA, Keene JD et al. Caspasemediated cleavage of HuR in the cytoplasm contributes to pp32/PHAP-I regulation of apoptosis. J Cell Biol 2008; 180: 113-127.

12. Fernando $P$, Kelly JF, Balazsi K, Slack RS, Megeney LA. Caspase 3 activity is required for skeletal muscle differentiation. Proc Natl Acad Sci USA 2002; 99: 11025-11030.

13. Huh MS, Parker MH, Scime A, Parks R, Rudnicki MA. Rb is required for progression through myogenic differentiation but not maintenance of terminal differentiation. $J$ Cell Biol 2004; 166: 865-876.

14. Porrello A, Cerone MA, Coen S, Gurtner A, Fontemaggi G, Cimino L et al. p53 regulates myogenesis by triggering the differentiation activity of pRb. J Cell Biol 2000; 151: 1295-1304.

15. Murray TV, McMahon JM, Howley BA, Stanley A, Ritter T, Mohr A et al. A non-apoptotic role for caspase-9 in muscle differentiation. J Cell Sci 2008; 121 (Pt 22): 3786-3793.

16. Yaffe $D$, Saxel $O$. Serial passaging and differentiation of myogenic cells isolated from dystrophic mouse muscle. Nature 1977; 270: 725-727.

17. Yaffe $D$, Saxel $O$. A myogenic cell line with altered serum requirements for differentiation. Differentiation 1977; 7: 159-166.

18. Huppertz B, Tews DS, Kaufmann P. Apoptosis and syncytial fusion in human placental trophoblast and skeletal muscle. Int Rev Cytol 2001; 205: 215-253.

19. Gallouzi IE, Brennan CM, Stenberg MG, Swanson MS, Eversole A, Maizels N et al. HuR binding to cytoplasmic mRNA is perturbed by heat shock. Proc Natl Acad Sci USA 2000; 97: 3073-3078.

20. Miura P, Thompson J, Chakkalakal JV, Holcik M, Jasmin BJ. The utrophin A $5^{\prime}$-untranslated region confers internal ribosome entry site-mediated translational control during regeneration of skeletal muscle fibers. J Biol Chem 2005; 280: 32997-33005.

21. Yan Z, Choi S, Liu X, Zhang M, Schageman JJ, Lee SY et al. Highly coordinated gene regulation in mouse skeletal muscle regeneration. J Biol Chem 2003; 278: 8826-8836.

22. Chipuk JE, Green DR. Cytoplasmic p53: bax and forward. Cell Cycle 2004; 3: 429-431.

23. Coletti D, Yang E, Marazzi G, Sassoon D. TNFalpha inhibits skeletal myogenesis through a PW1-dependent pathway by recruitment of caspase pathways. EMBO J 2002; 21: 631-642.

24. Rebane A, Aab A, Steitz JA. Transportins 1 and 2 are redundant nuclear import factors for hnRNP A1 and HuR. RNA 2004; 10: 590-599. 
25. Abdelmohsen K, Kuwano Y, Kim HH, Gorospe M. Posttranscriptional gene regulation by RNA-binding proteins during oxidative stress: implications for cellular senescence. Biol Chem 2008; 389: 243-255.

26. Dormoy-Raclet V, Menard I, Clair E, Kurban G, Mazroui R, Di Marco S et al. The RNAbinding protein HuR promotes cell migration and cell invasion by stabilizing the beta-actin mRNA in a U-rich-element-dependent manner. Mol Cell Biol 2007; 27: 5365-5380.

27. Fadeel B, Ottosson A, Pervaiz S. Big wheel keeps on turning: apoptosome regulation and its role in chemoresistance. Cell Death Differ 2008; 15: 443-452.
28. Yi $\mathrm{CH}$, Yuan J. The Jekyll and Hyde functions of caspases. Dev Cell 2009; 16: 21-34.

29. Brennan CM, Gallouzi IE, Steitz JA. Protein ligands to HuR modulate its interaction with target mRNAs in vivo. J Cell Biol 2000; 151: 1-14

30. Gramolini AO, Angus LM, Schaeffer L, Burton EA, Tinsley JM, Davies KE et al. Induction of utrophin gene expression by heregulin in skeletal muscle cells: role of the N-box motif and GA binding protein. Proc Natl Acad Sci USA 1999; 96: 3223-3227.

Supplementary Information accompanies the paper on Cell Death and Differentiation website (http://www.nature.com/cdd) 